Case Report

\title{
Massive Soft Tissue Haemophilic Pseudotumor in Limbs: Lessons Learnt from an Extremely Rare Problem
}

\section{F Munson* and A N Morritt}

Department of Plastic, Burns \& Reconstructive Surgery, Sheffield Teaching Hospitals, Sheffield, UK

\begin{tabular}{l} 
A R T I C L E I N F O \\
\hline Article history: \\
Received: 25 May, 2021 \\
Accepted: 9 June, 2021 \\
Published: 24 June, 2021 \\
\hline Keywords: \\
Haemophilic pseudotumor \\
soft tissue pseudotumor \\
surgical excision \\
case report
\end{tabular}

\begin{abstract}
A B S T R A C T
Haemophilic pseudotumors are very rare, encapsulated haematomas forms as a result of repetitive bleeding, forming a mass of clotted blood and necrosed tissue. Reported experience in the literature is limited to case reports and case series from around the world, reporting on smaller and medium sized pseudotumors. Conflicting opinion exists following failed medical management, which includes minimally invasive interventions through extensive surgery. We present our experience with the management of two of the largest soft tissue pseudotumors reported in the literature. We also provide some guidance, based on our experiences, for the future management of massive haemophilic pseudotumors.
\end{abstract}

\section{Introduction}

A haemophilic pseudotumor is an increasingly rare complication of haemophilia, especially in the developed world, affecting $1-2 \%$ of severe haemophiliacs (those with functional plasma levels of coagulation factor of $<1 \%$ ) worldwide. An encapsulated haematoma forms as a result of repetitive bleeding forming a mass of clotted blood and necrosed tissue. The trigger bleeding point is often a bone fracture site, a subperiosteal hemorrhage, or simply soft tissue trauma. With successive hemorrhagic episodes, these lesions expand over time, causing many of their symptoms by mass effect depending on anatomical site [1]. Reported experience in the literature is limited to case reports and case series from around the world. The limited documented experience also leads to a lack of consensus, and on occasion, contradictory opinion, on the best management of this debilitating and potentially life-threatening complication of haemophilia. Early surgical excision is advocated by some to prevent ulceration of the overlying skin, or underlying bone erosion [2]. Surgery has also been suggested as a recommended approach for pseudotumors that are of long-standing in which the body has failed to resorb the haematoma [3]. In addition, the literature pertains predominantly to small and medium-sized soft tissue limb pseudotumors. Understandably, the overriding concern related to surgery is the risk of uncontrollable hemorrhage.

The haemophilia service in Sheffield is world renowned and is a quaternary referral center for the management of haemophilia in the UK. In this capacity, two cases of this rare complication have been managed in the unit in recent years. In this paper, we present our experience with the surgical management of two of the largest pseudotumors reported in the literature. We also provide some guidance, based on our experiences, for the future management of massive haemophilic pseudotumors.

\section{Case Report}

\section{Case 1}

An 86-year-old male with severe haemophilia type B (0.8\% active clotting factor), replacement inhibitor negative, has managed

"Correspondence to: C F Munson, Department of Plastic, Burns \& Reconstructive Surgery, Sheffield Teaching Hospitals, Sheffield, UK; E-mail:

Chris.munson@doctors.org.uk 
haemophilia service in Sheffield for over 30 years. Approximately 15 years prior to this presentation, the patient developed a pseudotumor following hip replacement surgery. This had initially been managed conservatively in this period, simply with careful factor IX replacement. However, over recent years, he had persistent problems with a left thigh swelling (Figure 1). The swelling had reached a magnitude where concerns were raised about imminent ulceration and potential subsequent exsanguination. Despite internationally recognised experience in managing haemophilia, the Sheffield unit had not dealt with pseudotumors of this magnitude. Advice was sought from specialist units around the world, without consensus opinion. Magnetic Resonance Imaging revealed a 250mm x 130mm x 130mm pseudotumor (Figure 2).

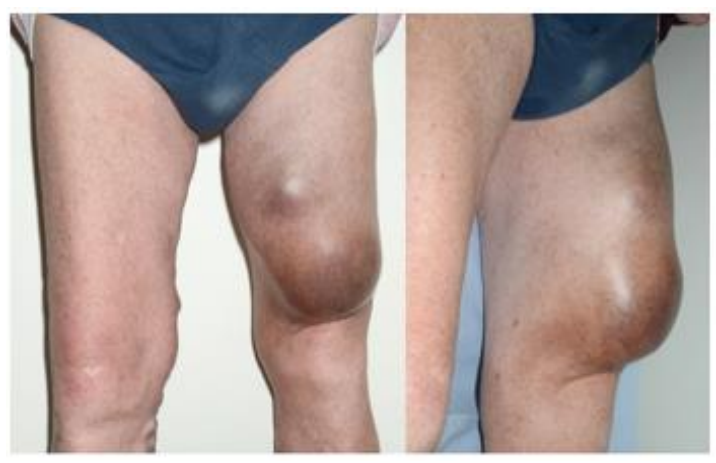

Figure 1: Left thigh pseudotumor patient 1 at time of presentation with threatened ulceration of overlying skin.

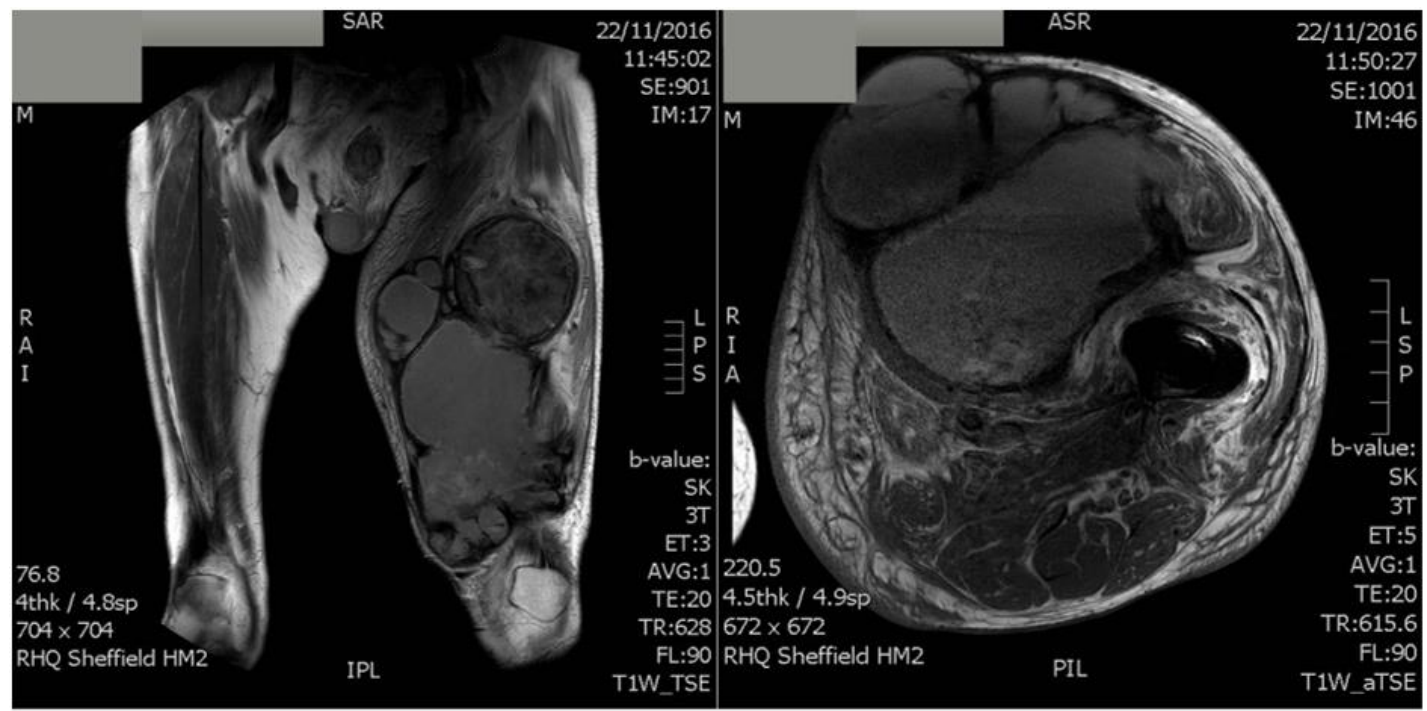

Figure 2: Patient 1 MRI. 250mm x 130mm x 130mm Pseudotumor within anterior compartment of the thigh.

Initial management was with an emergency, radiologically guided aspiration due to imminent ulceration of overlying skin (Figure 3). Unfortunately, haematoma reformed within 24 hours, and the patient developed a severe infection, progressing to systemic sepsis. The Plastic Surgery service in Sheffield was then asked to intervene in management of the infected haematoma. A surgical evacuation was performed. 3000 milliliters of haematoma were evacuated, no distinct bleed points identified, and a stabilising negative pressure dressing applied to allow further stabilisation of the patient. 48 hours later, the patient had improved sufficiently to allow further exploration, insertion of negative pressure drains, and closure of wounds. Again, however, reaccumulation of the haematoma occurred, with accompanying wound dehiscence. Despite optimal haematological input with factor VIII replacement and blood product transfusion, bleeding continued, necessitating a third procedure with further evacuation of haematoma, excision of the anterior wall of the pseudotumor, Argon cautery and the liberal application of haemostatic granules. Again, within one-week haematoma reaccumulated. A fourth procedure was planned by the senior author, with multidisciplinary input from the local soft tissue sarcoma, orthopaedic and haemophilia teams. A functional anterior compartmentectomy, preserving part of vastus lateralis and the femoral nerve was performed without intraoperative complication. A topical negative dressing was applied.

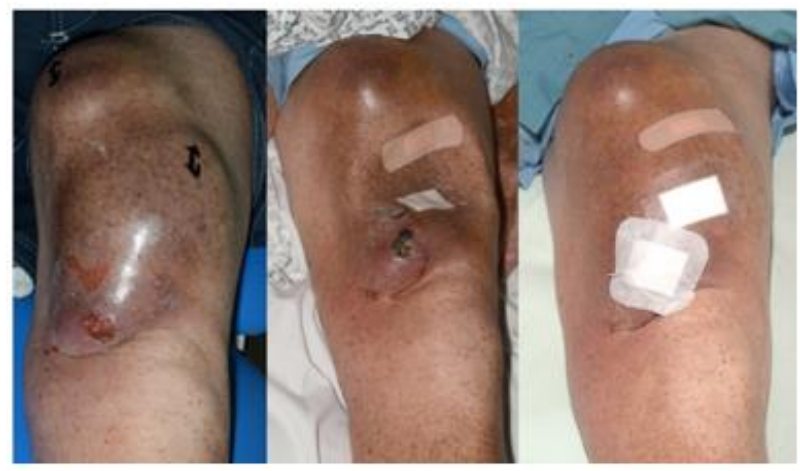

Figure 3: Prior to aspiration (left), immediately following aspiration (center) and re-accumulation 24 hours following aspiration (right).

The patient returned to the intensive care unit and made good postoperative progress, with no further bleeding or re-accumulation of haematoma. Unfortunately, the patient developed a hospital acquired pneumonia and passed away one month following his final operative procedure.

\section{Case 2}

Following the experience from the above case, the second described case was approached in a different fashion. A 39-year-old student, originally 
from Nigeria, was referred to the Sheffield Haemophilia service with severe, untreated Haemophilia A and a symptomatic swelling in his left thigh (Figure 4). He was struggling to mobilise, had frequent pain, and difficulty in obtaining clothing that would fit over his thigh. Initial management from the haemophilia specialists involved stabilisation with factor VIII replacement. Whilst plasma levels returned to normal parameters; symptoms were not improved. This prompted a referral to the senior author for consideration of surgical excision. MRI revealed a pseudotumor extending from femoral neck to knee, wrapping around femoral nerve/vessels, sciatic nerve, onto the femur. The maximal dimensions were $260 \mathrm{~mm} \times 140 \mathrm{~mm} \times 130 \mathrm{~mm}$ (Figure 5). The patient was next referred to our interventional radiology service for pre-operative embolization, a technique employed for complex vascular malformations. However, angiogram revealed a complex system of small vessels, with an absence of feeding vessels large enough to embolize. The decision was therefore taken to proceed to surgical excision of the pseudotumor in a manner similar to that employed for the marginal excision of soft tissue sarcomas.

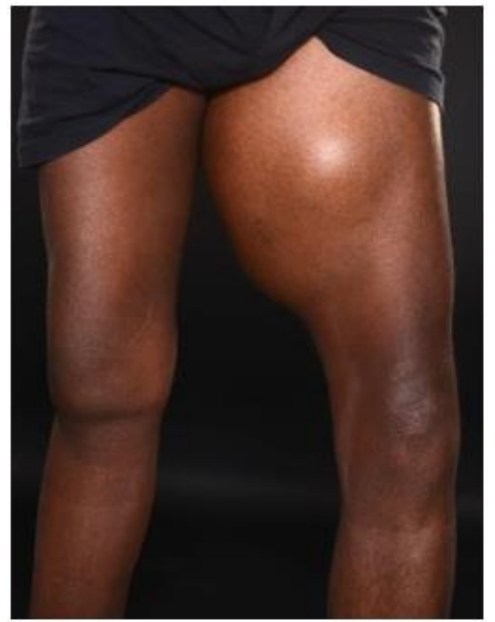

Figure 4: Left thigh pseudotumor of patient 2 at presentation.

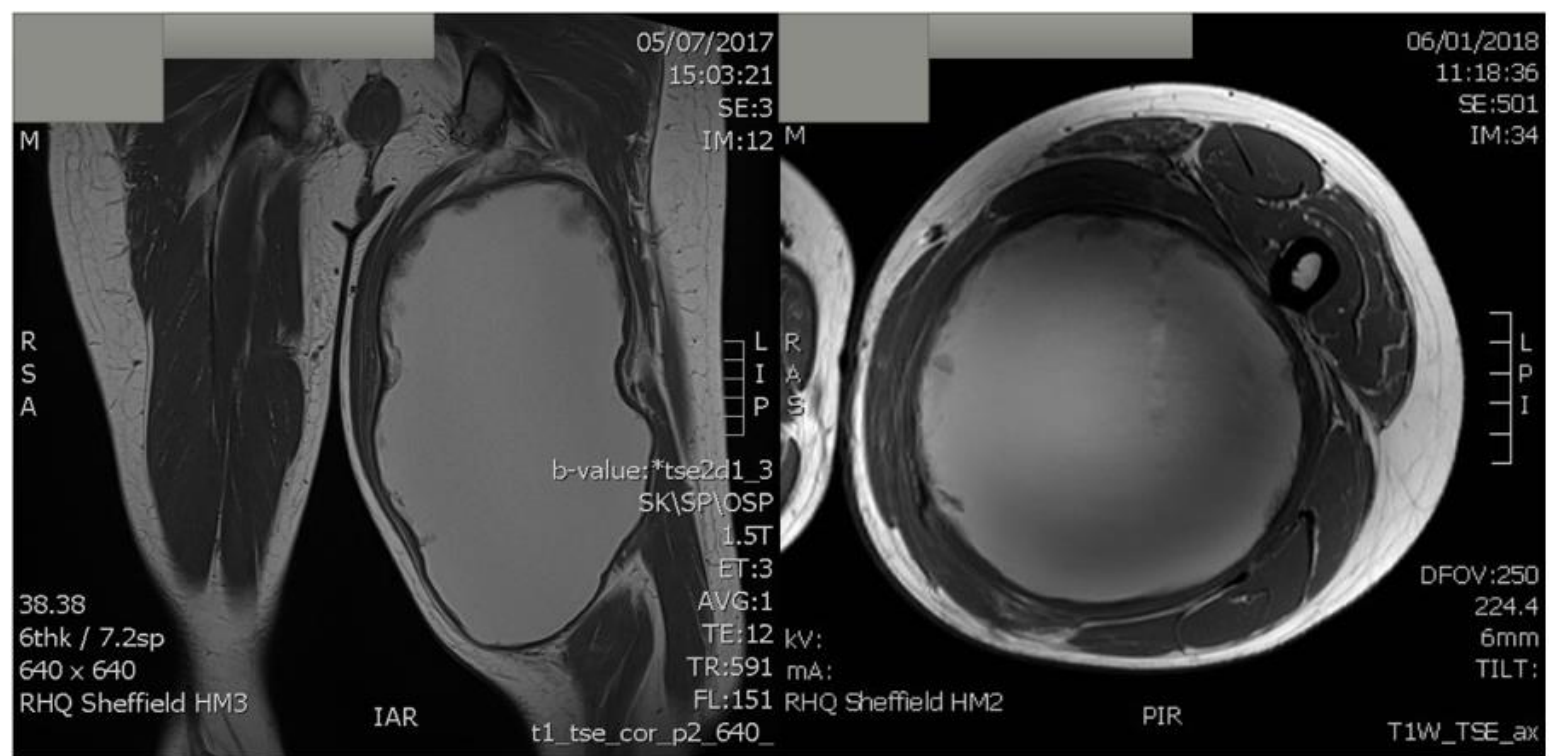

Figure 5: Patient 2 MRI. 260mm x 140mm x 130mm pseudotumor extending from femoral neck to knee, wrapping around femoral nerve/vessels, sciatic nerve, onto femur.

Pre-operative optimisation was performed with input from the Haemophilia service, anaesthetic, and critical care colleagues. Factor VIII replacement was run continually through the procedure and blood products available on stand-by. An anterior approach to the thigh was employed with a marginal excision of the pseudotumor (Figure 6). Once dissected clear from the femoral vessels and femoral nerve, the pseudotumor was decompressed of 2500 milliliters of haematoma in order to aid dissection from its superior, inferior, and posterior attachments, including the sciatic nerve. A sartorial switch procedure was performed to provide muscle cover for the femoral vessels so that they would not be exposed in the event of wound dehiscence. Two negative pressure percutaneous drains were inserted. Postoperative recovery was unremarkable. Factor VIII replacement continued until removal of both drains on seventh postoperative day. The patient was discharged on the eighth postoperative day. Subsequent follow-up shows no sign of recurrence two years following surgery. The patient is delighted with the result. He is pain free, mobilising without aid and now enjoys free sartorial choice!

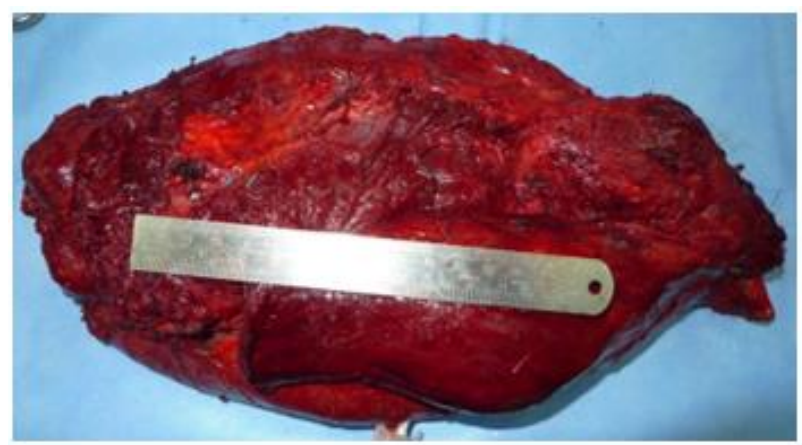

Figure 6: Excised pseudotumor from left thigh of patient 2.

\section{Discussion}

Haemophilic pseudotumors are a rare complication of severe haemophilia which was first described by Starker in the femur of a 14 year-old boy [4]. Three subtypes have been described: Proximal; 
affecting adults and related to long bones following trauma. It features bleeding into muscles adjacent to the bone and is frequently resistant to conservative management. Distal; seen most frequently in children related to the small bones of hands and feet. This type often responds to non-operative management. Finally, a third type, which arises from, and is limited to muscle with no bone involvement [5]. Patients may present with painless palpable masses or with painful crises due to episodic acute bleeding into the tumor. Most of the morbidity from pseudotumors is due to their compressive effect on the surrounding structures including bone destruction, muscle and skin necrosis, or nerve compression [6].

A conservative approach includes factor replacement and immobilisation of the affected area. This method has been suggested as the preferred management of pseudotumors caused by recent hemorrhage [3]. Minimal access drainage or aspiration of fluid within the cysts for either diagnostic or therapeutic purposes enjoys a mixed opinion, with some advocating it as the optimal management following a failed conservative approach [7]. Conversely, others consider it absolutely contraindicated [8]. Our experience certainly sits with the latter. It is likely that the chronic nature of the capsule means that complete collapse of the cavity following aspiration is unlikely, thus leading to re-accumulation and the potential to cause infection.

Surgery has been recommended by some early in the management of pseudotumors. Indications for surgery include clinical or radiographic evidence of progressive enlargement of the haematoma, haemodynamic deterioration or occurrence of a complication [4]. Factor replacement in combination with surgery has been shown to be more successful than medical management alone in one small series [8]. This is further supported in another case series that advocates the combined medical and surgical approach [9]. Aspiration followed by injection of fibrin or Spongostane ${ }^{\circledR}$ has also been reported, thus attempting to reduce the capacity of the cavity in the event of non-collapse of the capsule [7]. Therapeutic arterial embolization may be considered, especially for tumors of large size, which may result in reduction of the size of the tumor as well as reduction of bleeding complications during surgery. However, as evidenced in our second case, often feeding vessels are small, numerous and not amenable to embolization. Radiotherapy is a viable alternative where surgery is not feasible and conservative management is not effective. Around 6-23.5 Gy in fractions of 2 Gy or less has been found sufficient but has its own side effect profile including carcinogenesis and the potential to cause skin necrosis [10].

\section{Conclusion}

Our conclusions from managing two of the largest limb haemophilic pseudotumor found in the literature, can be summarised as follows: we strongly advocate a multidisciplinary approach, with specialist haematological input, critical care, and anaesthetic optimisation and radiological pre-operative planning (with the option of pre-operative embolization explored and employed if practicable). For these large chronic pseudotumors with rigid capsules, we recommend marginal excision of the whole pseudotumor and capsule. Where marginal excision is not possible due to the configuration of the pseudotumor e.g., extensive infiltration between muscles, functional compartmentectomy is advocated. The complete excision of the capsule appears to be a key component in successful treatment and conversely, repeated aspiration or only partial capsulectomy incurs a high risk of both recurrence and further complications including infection, skin necrosis and wound dehiscence.

\section{Acknowledgement}

We would like to acknowledge the input of the following members of the haematology and anaesthetic departments at Sheffield Teaching Hospitals: Professor M Makris, Dr. R Maclean, Dr. J Van Veen, Dr. I Shaw.

\section{REFERENCES}

1. Pakala A, Thomas J, Comp P (2012) Hemophilic Pseudotumor: A Case Report and Review of Literature. Int J Clin Med 3: 229-233.

2. Rosenthal RL, Graham JJ, Selirio E (1973) Excision of pseudotumor with repair by bone graft of pathological fracture of femur in hemophilia. J Bone Joint Surg Am 55: 827-832. [Crossref]

3. Gaary E, Gorlin JB, Jaramillo D (1996) Pseudotumor and arthropathy in the knees of a hemophiliac. Skeletal Radiol 25: 85-87. [Crossref]

4. Starker L (1918-1919) Knochenusur durch ein hamophiles, subperiosteal Hematoma. Mitt Med Chir 31: 381.

5. Gilbert MS (1975) Characterizing the hemophilic pseudotumor. Ann NY Acad Sci 240: 311-315. [Crossref]

6. Park JS, Ryu KN (2004) Hemophilic pseudotumor involving the musculoskeletal system: spectrum of radiologic findings. AJR Am J Roentgenol 183: 55-61. [Crossref]

7. Cavaiglia H, Landro ME, Galatro G, Candela M, Neme D (2015) Pseudotumors of the Limbs in Patients with Hemophilia. J Blood Disord 2: 1026.

8. Magallón M, Monteagudo J, Altisent C, Ibanez A, Rodriguez Perez A et al. (1994) Hemophilic pseudotumor: multicenter experience over a 25-year period. Am J Hematol 45: 103-108. [Crossref]

9. Zhai J, Weng X, Zhang B, Liu Y, Gao P et al. (2017) Surgical Treatment for Hemophilic Pseudotumor: Twenty-three Cases with an Average Follow-up of 5 Years. J Bone Joint Surg Am 99: 947-953. [Crossref]

10. Castaneda VL, Parmley RT, Bozzini M, Feldmeir JJ (1991) Radiotherapy of pseudotumors of bone in hemophiliacs with circulating inhibitors to factor VIII. Am J Hematol 36: 55-59. [Crossref] 